\title{
No a la guerra: resistencia civil en dos comunidades periféricas de Colombia*
}

\author{
CÉCILE MOUlY** \\ MARÍA BELÉN GARRIDO***
}

Artículo recibido: 5 de diciembre de 2016

Artículo aprobado: 24 de julio de 2017

Doi: http://dx.doi.org/10.12804/revistas.urosario.edu.co/desafios/a.5282

Para citar este artículo: Mouly, C., \& Garrido, M.B. (2018). No a la guerra: resistencia civil en dos comunidades periféricas de Colombia. Desafíos, 30(1), 245-277. Doi: http://dx.doi. org/10.12804/revistas.urosario.edu.co/desafios/a.5282

\section{Resumen}

Este artículo compara el accionar de dos comunidades que se declararon "territorio de par" en Colombia: el municipio de Samaniego, en el departamento suroccidental de Nariño, fronterizo con Ecuador, y el corregimiento de Las Mercedes, en el departamento oriental de Norte de Santander, fronterizo con Venezuela. Estas comunidades comparten una situación similar. Fueron fuertemente afectadas por la

* Este artículo se basa en un artículo titulado "Peace Territories in Colombia: Comparing Civil Resistance in Two War-Torn Communities”, publicado en inglés en 2015 (Idler, Garrido \& Mouly, 2015), y en una ponencia presentada en el VIII Congreso Latinoamericano de Ciencia Política, organizado por la Asociación Latinoamericana de Ciencia Política (ALACIP) en julio de 2015.

** Ph.D. en Estudios Internacionales, Universidad de Cambridge. Profesora investigadora, Facultad Latinoamericana de Ciencias Sociales (FLACSO), Quito, Ecuador. Correo electrónico: camouly@flacso.edu.ec. ORCID: http://orcid.org/0000-0002-0499-8213

*** Estudiante de doctorado de la Universidad Católica de Eichstaett-Ingolstadt, Alemania. Máster en Estudios de Paz, FernUniversitaet en Hagen, Alemania. Investigadora, Flacso Quito, Ecuador. Profesora investigadora, Pontificia Universidad Católica del Ecuador, Quito, Ecuador. Correo electrónico: mbgarrido@flacso.edu.ec. oRCID: http://orcid. org/0000-0002-2496-7561 
guerra y se volvieron lugares de cultivos de coca. Frente a esta situación la población civil emprendió un proceso de resistencia no-violenta en contra de actores armados estatales y no estatales. Usamos una metodología cualitativa, que incluyó entrevistas, observación y análisis de fuentes documentales para comparar los resultados de estas acciones en ambos casosy entender las razones de su éxito o fracaso. Encontramos que tres tipos de razones pueden explicar los distintos resultados de estas acciones: i) las características del movimiento de resistencia civil, incluyendo la participación en el movimiento, su cohesión y su lideraggo; ii) las relaciones entre la comunidady los grupos armados, y iii) el respaldo de actores externos. Finalmente, argumentamos que las negociaciones de paz a nivel nacional pueden potenciar las iniciativas locales de pazy que estas son importantes para la paz en Colombia.

Palabras clave: resistencia civil, no-violencia, conflicto armado interno, sociedad civil, construcción de par.

\title{
No to War: Civil Resistance in Two Peripheral Communities of Colombia
}

\begin{abstract}
This article compares the actions of two communities that declared themselves "peace territory" in Colombia: the municipality of Samaniego in the southwestern department of Narino, bordering Ecuador, and the town ("corregimiento") of Las Mercedes in the eastern department of Norte de Santander, bordering Venezuela. These communities share a similar situation. They were strongly affected by war and became places of coca cultivation. In the face of this situation the civilian population undertook a process of nonviolent resistance against state and non-state armed actors. We use a qualitative methodology, including interviews, observation and the analysis of documentary sources, to compare the outcomes of these actions in both cases and understand the reasons of their success or failure. We found that three types of reasons can explain the distinct outcomes of these actions: $i)$ the characteristics of the civil resistance movement, including the participation in the movement, its cohesion and its leadership, ii) the relationships between the community and armed groups, and iii) the support of external actors. Finally, we argue that peace negotiations at the national level can foster local peace initiatives, and that these are important for peace in Colombia.
\end{abstract}

Keywords: Civil resistance, nonviolence, armed conflict, civil society, peacebuilding. 


\title{
Não à guerra: resistência civil em duas comunidades periféricas da Colômbia
}

\begin{abstract}
Resumo
Este artigo compara o acionar de duas comunidades que se declararam "território de paz" na Colômbia: o município de Samaniego no departamento sul-ocidental de Nariño, fronteiriço com o Equador, e o corregimento de Las Mercedes no departamento oriental de Norte de Santander, fronteiriço com a Venezuela. Estas comunidades compartilham uma situação similar. Foram fortemente afetadas pela guerra e voltaram-se lugares de cultivos de coca. Frente a esta situação a população civil empreendeu um processo de resistência não-violenta contra os atores armados estatais e não estatais. Usamos uma metodologia qualitativa, incluindo entrevistas, observação e a análise de fontes documentais, para comparar os resultados destas ações em ambos os casos e entender as razõos de seu sucesso ou fracasso. Encontramos que três tipos de razões podem explicar os distintos resultados destas ações: i) as caracteristicas do movimento de resistência civil, incluindo a participação no movimento, sua coesão e sua liderança, ii) as relaçoes entre a comunidade e os grupos armados e iii) o respaldo de atores externos. Finalmente, argumentamos que as negociações de paz no nivel nacional podem potenciar as iniciativas locais de paz. que estas são importantes para a paz na Colômbia.
\end{abstract}

Palavras-chave: resistência civil, não-violência, conflito armado interno, sociedade civil, construção de pa\%.

\section{Introducción}

Los esfuerzos locales por construir la paz en lugares inmersos en conflictos armados han llamado la atención de muchos académicos, especialmente en Colombia (ej. Hernández, 2004, 2012; Sanford, 2003; Rojas, 2004, 2007). Entre estos, se encuentran experiencias de bases como las comunidades o territorios de paz (Alther, 2006) o a nivel regional como los programas de desarrollo y paz (García \& Llinás, 2012). También existen experiencias basadas en identidades étnicas, como las del Consejo Regional Indígena del Cauca o del Consejo Comunitario Mayor de la ACIA (Hernández, 2012). Estas experiencias tienen en común el uso de métodos no-violentos para 
reducir tanto las manifestaciones violentas del conflicto armado como sus raíces estructurales que se encuentran, entre otras, en la marginación socioeconómica de estos territorios. Como veremos más adelante, varios estudios sobre construcción de paz a nivel local (ej. Lederach, 1997; Mitchell \& Hancock, 2012) muestran cómo estos esfuerzos pueden contribuir a la paz a nivel nacional. En el marco de las negociaciones de paz entre el Gobierno colombiano y las Fuerzas Armadas Revolucionarias de Colombia (FARC), que iniciaron en el año 2012, se introdujo el concepto de paz territorial para recalcar la necesidad de concebir la construcción de paz, no solamente como un proceso impulsado desde el nivel central, sino también desde las comunidades que viven a lo ancho del territorio ${ }^{1}$. En este contexto es menester estudiar los esfuerzos locales de paz que se han dado en Colombia y entender las razones de sus éxitos y fracasos para potenciarlos. Solo así se puede construir una paz incluyente que abarque la diversidad territorial y garantizar una apropiación del proceso por parte de algunas de las comunidades que más han sufrido los efectos del conflicto armado colombiano.

Este artículo reflexiona en torno a las experiencias de dos comunidades colombianas que han intentado establecerse como territorios de paz al fijar ciertas reglas del juego frente al conflicto armado en su territorio. Además, trata de entender los factores que han incidido en el éxito o las limitaciones de las acciones de resistencia civil de la población contra los actores armados presentes en su territorio. Calificamos de "exitosas" las acciones de resistencia civil que provocaron un cambio en los actores hacia los cuales estaban dirigidas, como, por ejemplo, un mayor respeto de la población civil por parte de los actores armados luego de una protesta en contra de los abusos

\footnotetext{
1 El Alto Comisionado para la Paz de Colombia introdujo el término de paz territorial (Jaramillo, 2014). Este enfoque se ve reflejado en el Acuerdo final para la terminación del conflicto y la construcción de una paz estable y duradera, firmado por el Gobierno colombiano y las FARC en 2016. Por ejemplo, en la introducción se menciona que "[e]l enfoque territorial del Acuerdo supone reconocer y tener en cuenta las necesidades, características y particularidades económicas, culturales y sociales de los territorios y las comunidades" (Acuerdo final para la terminación del conflicto y la construcción de una paz estable y duradera, 2016, p. 6).
} 
perpetrados por estos actores. Las dos comunidades están situadas respectivamente en el municipio de Samaniego, en el departamento de Nariño, fronterizo con Ecuador, y en el corregimiento de Las Mercedes, ubicado en el municipio de Sardinata, en el departamento de Norte de Santander, fronterizo con Venezuela. Ambas comunidades han buscado mantenerse imparciales en medio del conflicto armado y han usado métodos no-violentos para persuadir a los actores armados estatales y no estatales de respetar ciertas reglas del juego en su territorio y no atacar a la población e infraestructura civil, lo que les ha valido ser referidas como territorios de paz, un término usado con frecuencia en Colombia y equivalente al de zona de paz, más usado en la academia (Hancock \& Mitchell, 2007; Mitchell \& Allen Nan, 1996) ${ }^{2}$. En este artículo, definimos territorio de paz como territorio en el que la población civil se considera imparcial en el conflicto armado interno y establece reglas de juego para limitar las consecuencias negativas de la guerra.

Este artículo se basa en trabajo de campo realizado en Samaniego y Las Mercedes y las ciudades colombianas de Bogotá, Cali, Pasto y Cúcuta entre los años 2014 y 2016, con más de 100 entrevistas, observación in situ y el análisis de documentos. Las autoras realizaron entrevistas a diversos actores, que incluyeron integrantes de los movimientos de resistencia civil de ambas comunidades, autoridades locales, miembros de organizaciones externas (nacionales e internacionales), líderes religiosos y miembros y exmiembros de grupos armados estatales y no estatales que operaron en Samaniego o Las Mercedes ${ }^{3}$. El análisis de los dos casos de estudio corre desde finales de los años noventa hasta mediados de 2016 y se centra en los periodos de mayor movilización en contra de la violencia perpetrada

\footnotetext{
2 El término territorio de paz ganó trascendencia a raíz del proyecto Cien municipios de paz, implementado por la Red Nacional de Iniciativas Ciudadanas por la Paz y contra la Guerra (Redepaz) a finales de los años noventa.

3 En Samaniego se realizaron aproximadamente 19 entrevistas a exfuncionarios del Estado, 11 a exmiembros de organismos internacionales, 20 expobladores, 9 a exactores armados y 30 a exmiembros de organizaciones de la sociedad civil colombiana. En Las Mercedes se realizaron aproximadamente 10 entrevistas a exfuncionarios del Estado, 5 a exmiembros de organismos internacionales, 13 pobladores o expobladores, 2 a exactores armados y 10 a exmiembros de organizaciones de la sociedad civil colombiana.
} 


\section{Mapa 1. Ubicación de Samaniego y Las Mercedes}

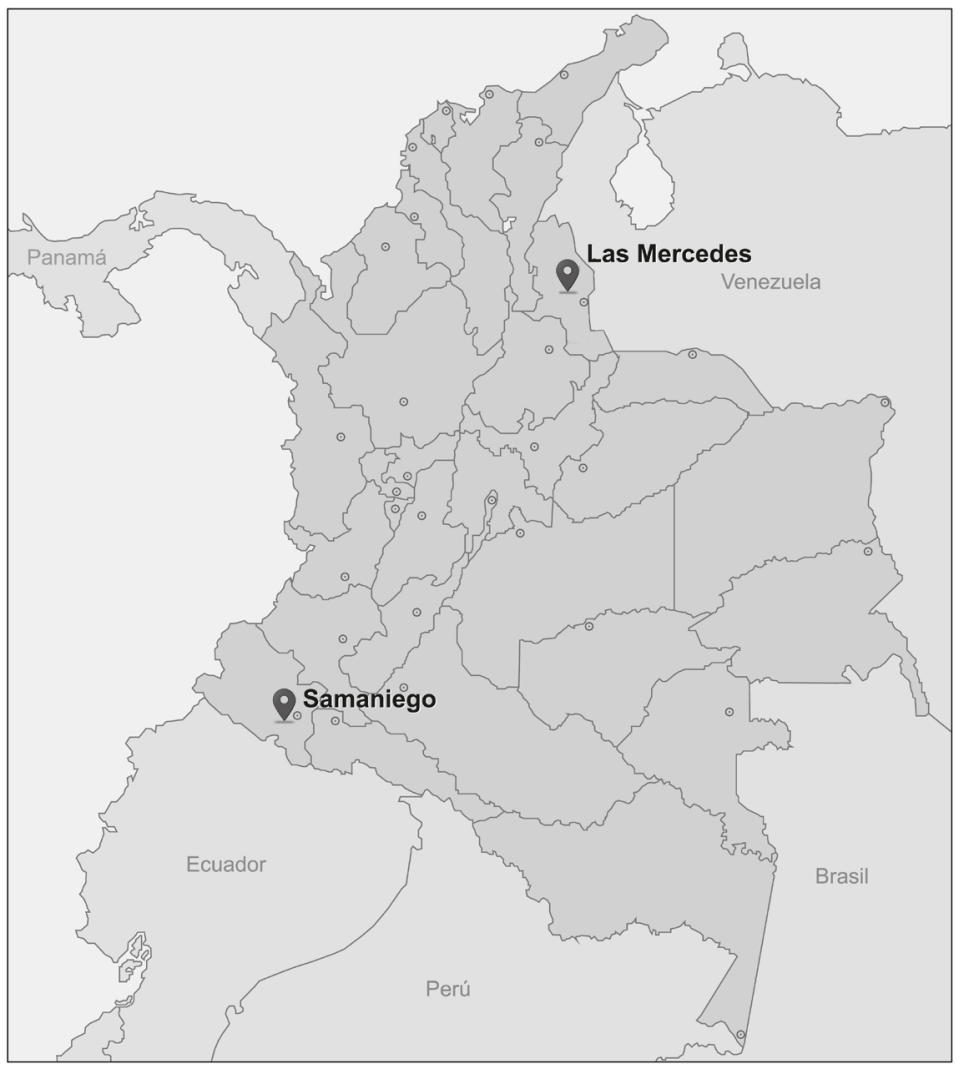

Fuente: Mouly, Idler \& Garrido (2015).

por los actores armados, aunque también analiza las razones del declive del proceso de resistencia civil en ciertos periodos.

El artículo se divide en tres partes. En la primera parte se presenta el contexto en que viven ambas comunidades y las estrategias noviolentas utilizadas por ellas para reducir el impacto negativo del conflicto armado en ambas localidades. En la segunda parte se discuten los efectos que tres grupos de factores tuvieron sobre los resultados de acciones de resistencia civil emprendidas en Samaniego y Las Mercedes a partir de una comparación entre ambas experiencias. En 
la tercera parte se examina la incidencia de la construcción de paz a nivel nacional sobre estas iniciativas locales de paz y cómo estas últimas pueden aportar a la paz en Colombia.

\section{Samaniego y Las Mercedes: territorios de paz en medio de un conflicto armado}

Samaniego y Las Mercedes comparten un contexto común. Tanto Samaniego como Las Mercedes están ubicados en departamentos fronterizos marginados que han sido fuertemente golpeados por el conflicto armado. Sus poblaciones han sido afectadas por el narcotráfico y sus posiciones geográficas han hecho que sean corredores estratégicos para este, ya sea a través del Pacífico hacia Estados Unidos o por el Caribe hacia Europa o Estados Unidos. Históricamente, las dos localidades han sido relegadas por el Gobierno central y han sufrido las consecuencias de la llamada política de seguridad democrática impulsada por el expresidente Álvaro Uribe, la cual se enfocó en mejorar la seguridad en grandes ciudades como Bogotá, pero afectó las periferias e hizo que el conflicto se trasladara a espacios donde la presencia estatal era limitada o nula, como Samaniego y Las Mercedes (Mitchell \& Rojas, 2012). Los niveles de pobreza en ambas comunidades han sido altos ${ }^{4}$. El transporte público, la infraestructura de telecomunicaciones y los servicios básicos han sido deficientes en comparación con muchos lugares que comparten características geográficas y políticas similares en Colombia 5 .

De igual forma, ambos territorios han tenido una fuerte presencia de grupos insurgentes como las FARC y el Ejército de Liberación Nacional (ELN) y sufrieron incursiones de grupos paramilitares. En Samaniego,

\footnotetext{
4 En 2013 el 58,03\% de personas tenía necesidades básicas insatisfechas (en el área rural este porcentaje sube a alrededor de 70\%) en Samaniego, y el 53,30\% de personas tenía necesidades básicas insatisfechas en Sardinata. Estas cifras representan aproximadamente el doble del promedio nacional que es de 27,67\% (Criollo \& Castillo, 2013; Yanes, 2013).

5 En 2013, en el área rural de Samaniego, la cobertura de acueducto era del 58\%, electricidad del $87 \%$ y alcantarillado del 4,9\%. En el área rural de Sardinata, que incluye Las Mercedes, la cobertura de acueducto era del 18,53\%, electricidad del 55,56\% y alcantarillado del 17,82\%. La vía de acceso a Las Mercedes estaba en mal estado y la vía de acceso a Samaniego en estado regular (Criollo \& Castillo, 2013; Yanes, 2013).
} 
las FARC y el ELN han tenido presencia en el área rural desde finales de los años ochenta. Desde entonces las dos guerrillas han incidido en el municipio, pero el ELN ha tenido mayor influencia en la gestión municipal, ejerciendo control en gran parte del sector llamado de la "montaña", mientras las FARC controlaron zonas menos pobladas. A inicios del 2000, los paramilitares ingresaron a Samaniego disputando el control a las guerrillas hasta la desmovilización del Bloque Libertadores del Sur de las Autodefensas Unidas de Colombia (AUC), en 2005. Al finalizar el año 2006, las FARC y el ELN empezaron a enfrentarse en el territorio hasta alcanzar un acuerdo a finales de 2009. Desde entonces, hasta la desmovilización de las FARC en 2016, las dos guerrillas mantuvieron control sobre sus áreas respectivas del territorio, con el ELN nuevamente como actor armado dominante en el municipio (Mouly, Garrido \& Idler, 2016).

En Las Mercedes, las guerrillas también ingresaron en los años ochenta. Las dos guerrillas más fuertes en ese entonces eran las FARC y el ELN, pero el Ejército Popular de Liberación (EPL) también actuaba en el área y en 1998 las tres guerrillas lanzaron una ofensiva conjunta contra la Policía que dejó a la comunidad sin estación de Policía por varios años. La primera incursión paramilitar ocurrió en 2001 cuando el Bloque Catatumbo de las AUC empezó a desafiar el dominio de las guerrillas; el territorio estuvo en disputa hasta la desmovilización del bloque en 2004-2005. Posteriormente, las FARC volvieron a asumir el control de Las Mercedes hasta su desmovilización, con el ELN y el EPL ejerciendo control en áreas aledañas e inclusive en algunas veredas de Las Mercedes (Idler, Mouly \& Garrido, por publicar).

Ambas localidades son estratégicas para los actores armados, quienes buscan controlar el territorio y explotar recursos lucrativos. Las áreas rurales de Samaniego y Las Mercedes están pobladas principalmente por campesinos que dependen de la economía de la coca, debido a las limitadas oportunidades legales de subsistencia ${ }^{6}$. La presencia de grupos armados en la región ocasionó una migración en la población.

\footnotetext{
6 En 2013 el municipio de Sardinata tenía un índice ruralidad del 60\%, mientras Samaniego tenía un índice de ruralidad del $40 \%$. En ambos casos la población indígena y afrodescendiente era menor al 2\% (Criollo \& Castillo, 2013; Yanes, 2013).
} 
Algunas personas llegaron para trabajar en la producción de la coca o la explotación minera, mientras otras escaparon de la violencia.

Frente a la situación de conflicto armado, las dos comunidades emprendieron un proceso de resistencia civil, entendida como la aplicación de métodos no-violentos y no convencionales contra oponentes que tienen un poder coercitivo superior y están dispuestos a usar la violencia para imponerse (Chenoweth \& Cunningham, 2013; Schock, 2013). Este proceso llevó a ambas a establecerse como territorios de pa\%. El municipio de Samaniego fue declarado territorio de paz en 1998 bajo el impulso del entonces alcalde Manuel Cuéllar con el apoyo mayoritario de la población (Diario del Sur, 1998a). Posteriormente, en 2004, el alcalde Harold Montúfar planteó un pacto local de paz con los grupos armados presentes en Samaniego, que consistía en diez puntos que estos grupos debían respetar (ver cuadro 1). Mientras tanto, en 2005, un grupo de líderes, con el apoyo de su comunidad, impulsó el establecimiento de un territorio libre de acciones armadas en el corregimiento de Las Mercedes mediante una declaración de convivencia pacífica (ver cuadro 2), difundida ampliamente y dada a conocer a los actores armados estatales y no estatales (Las Mercedes, 2005; Peñaloza, 2006).

Cuadro 1. Diez puntos del pacto local de paz de Samaniego

\begin{tabular}{|l|}
\hline 1. Declarar la neutralidad, autonomía e imparcialidad frente al conflicto armado. \\
\hline 2. Exigir el respecto a la vida, a la sociedad civil y a no ser parte de la guerra. \\
\hline 3. Construir localmente un Estado social de derecho con justicia social. \\
\hline 4. No reconocer la guerra y la violencia como método para resolver los conflictos. \\
\hline 5. Respetar la diversidad de pensamientos, culturas y creencias. \\
\hline 6. Apoyar la solución política negociada al conflicto armado. \\
\hline 7. Exigir el respeto de los derechos humanos y del derecho internacional humanitario. \\
\hline $\begin{array}{l}\text { 8. Reclamar que no haya obstrucción a la circulación de los bienes básicos, ni } \\
\text { destrucción de infraestructura física, ni de los espacios culturales. }\end{array}$ \\
\hline $\begin{array}{l}\text { 9. Reivindicar de los grupos armados el cese de hostilidades en los espacios culturales: } \\
\text { concurso departamental y nacional de bandas musicales, carnaval de Samaniego, } \\
\text { semana cultural, juegos deportivos campesinos, festival de música campesina, fiestas de } \\
\text { San Martín, aniversario de Samaniego. }\end{array}$ \\
\hline $\begin{array}{l}\text { 10. Exigir el respeto de los territorios de paz: escuelas, colegios, casa campesina, } \\
\text { instituto de cultura, residencias estudiantiles, alcaldía municipal y acueducto municipal. }\end{array}$ \\
\hline
\end{tabular}

Fuentes: Lázaro Méndez (2010); Montúfar (2007). 


\section{Cuadro 2. Seis puntos de la declaración de convivencia pacífica de Las Mercedes}

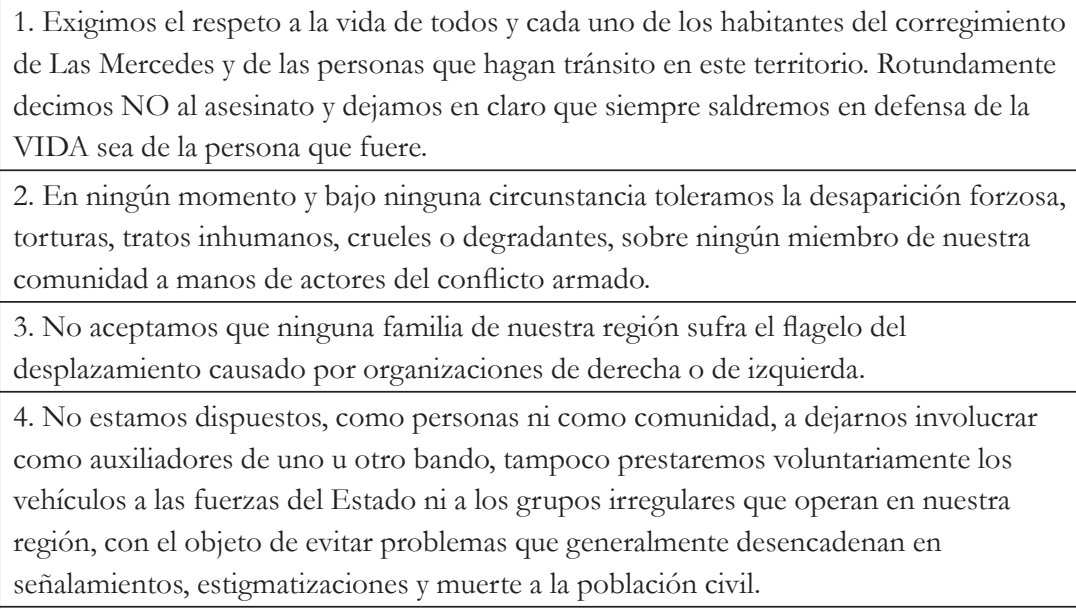

2. En ningún momento y bajo ninguna circunstancia toleramos la desaparición forzosa, torturas, tratos inhumanos, crueles o degradantes, sobre ningún miembro de nuestra comunidad a manos de actores del conflicto armado.

3. No aceptamos que ninguna familia de nuestra región sufra el flagelo del desplazamiento causado por organizaciones de derecha o de izquierda.

4. No estamos dispuestos, como personas ni como comunidad, a dejarnos involucrar como auxiliadores de uno u otro bando, tampoco prestaremos voluntariamente los vehículos a las fuerzas del Estado ni a los grupos irregulares que operan en nuestra región, con el objeto de evitar problemas que generalmente desencadenan en señalamientos, estigmatizaciones y muerte a la población civil.

5. Los comerciantes, empleados, peseros, dueños de vehículos, ganaderos, profesores, agricultores, ni miembro alguno de nuestra comunidad está en condición ni tiene la capacidad de aportar cuotas económicas que financien en lo más mínimo la guerra a ningún actor del conflicto existente en esta región. Tampoco consideramos una obligación los aportes en especies.

Aclaramos que con lo anterior nuestra intención no es la de estar a favor o en contra de organismo u organización alguna, respetamos las diferencias aunque no compartamos ideales y, como sociedad civil que busca y se compromete con la PAZ, a nadie le podemos prohibir la presencia civil en nuestro terruño agradeciendo no instalarse permanente [sic] en el centro poblado.

6. La comunidad de Las Mercedes se compromete públicamente a elaborar un plan de desarrollo social y económico del corregimiento, para trabajar ordenada y además muy responsablemente par[a] satisfacer nuestras necesidades con primacía del bien común.

Fuente: Las Mercedes (2005).

Como se puede observar en estas declaraciones, las poblaciones de Samaniego y Las Mercedes han exigido a los actores armados de los distintos bandos presentes en su territorio un mayor respeto a los civiles mediante el cumplimiento de reglas específicas. Al mismo tiempo, han planteado su no involucramiento como población civil en la guerra. Si bien el tono de las dos declaraciones es distinto y cada una contiene particularidades propias del contexto local, las dos fueron inicialmente bien recibidas por los actores armados y produjeron algunos resultados, tal como se discute a continuación. 


\section{Samaniego y Las Mercedes: en búsqueda de la paz en sus territorios}

A pesar de que Samaniego y Las Mercedes tienen un contexto similar, han alcanzado resultados diferentes en sus procesos de resistencia civil. En Samaniego el proceso de resistencia civil tuvo éxito en buena medida durante el periodo de estudio y permitió evitar en gran parte que los grupos armados involucraran a la población civil y atacaran la infraestructura civil. Mientras tanto, en Las Mercedes, esfuerzos similares fueron exitosos al inicio pero decayeron posteriormente y volvieron a tomar impulso después del inicio de las negociaciones de paz entre el gobierno de Juan Manuel Santos y las FARC, a finales de 2012. En su estudio sobre zonas de paz, Landon Hancock y Christopher Mitchell (2007) sugieren varios factores que pueden ayudar a explicar estos diferentes resultados, incluyendo: i) la unidad de propósito del movimiento de resistencia civil; ii) el desarrollo de una variedad de acciones que van más allá de la búsqueda del cese de la violencia directa; iii) un liderazgo colectivo aceptado por la comunidad; iv) la imparcialidad frente al conflicto, y v) el apoyo de actores externos. De la misma forma, Mary Anderson y Marshall Wallace (2013) proponen elementos que contribuyen al éxito de lo que llaman comunidades de no guerra (nonwar communities en inglés), otro término equivalente al de territorios de paz en Colombia. Estos elementos incluyen la cohesión de la comunidad, el liderazgo compartido, la capacidad de interlocución con actores armados y el apoyo de actores externos. Agrupamos los factores identificados por estos dos grupos de autores en tres categorías: en primer lugar, los que se refieren a las características del movimiento de resistencia civil; en segundo lugar, los que tienen que ver con la relación de la comunidad con los grupos armados, y en tercer lugar, los vinculados al rol de los actores externos.

\section{Características del movimiento de resistencia civil}

Los factores clave que se refieren a características del movimiento de resistencia civil son: su cohesión, la participación masiva de la población y el liderazgo que debe ser apoyado por la población 
en general. Al momento de la declaración como territorio de paz, Samaniego se encontraba bajo la fuerte presencia y control del ELN. Durante la campaña electoral de 1997, este grupo armado secuestró al candidato a alcalde, Manuel Cuéllar, y lo obligó a renunciar a su candidatura, pero los habitantes de Samaniego se organizaron y presionaron por su liberación y el mantenimiento de su candidatura. Además, votaron masivamente por él en las elecciones. Apenas fue electo y asumió sus funciones, Cuéllar invitó a la población a participar en el establecimiento del municipio como territorio de paz. Este esfuerzo se caracterizó por la unidad de la comunidad y su apoyo masivo a la declaración de territorio de paz y resultó en la aceptación de la iniciativa por parte del grupo armado dominante mediante un comunicado (Diario del Sur, 1997a, 1997b, 1998a, 1998b, 1998c; El Tiempo, 1997a, 1997b, 1997c; Rojas, 2004, p. 10). En efecto, la unidad de un movimiento de resistencia civil es clave para su éxito. Así, en su estudio de ochenta campañas de resistencia civil, Chenoweth y Stephan (2011, p. 44) encontraron, tal como es de esperar, que era más difícil derrotar un movimiento de resistencia civil unido. De forma parecida, Anderson y Wallace (2013, p. 56) argumentan que la cohesión de una comunidad permite resistir a las presiones externas.

En Las Mercedes, en el año 2005, luego de algunos años de arremetida paramilitar y abusos de parte de los actores armados en pugna, un grupo de líderes comunitarios autodenominado "Amigos por la paz" organizó reuniones informales e intentó convencer a otros miembros de la comunidad de unirse a sus esfuerzos para paliar los efectos negativos de la guerra. Este esfuerzo dio lugar a la elaboración de una declaración de convivencia pacífica. En esta declaración, avalada por la comunidad, se demandó a los grupos armados no involucrar a la población civil en el conflicto armado. Así se logró que estos dejaran de realizar ataques en el casco urbano en un inicio (Las Mercedes, 2005; entrevistas con pobladores y actores externos, 2014-2016). Según un excomandante de las FARC, el respaldo de toda la comunidad a la iniciativa fue una de las principales razones de su éxito inicial (entrevista con excomandante de las FARC 2016). Por ejemplo, en una ocasión, unos guerrilleros llegaron al pueblo para usar las motos de unos habitantes. Unos líderes de Amigos por la paz, 
con el apoyo de la comunidad, lograron impedir que los guerrilleros se las llevasen. Al final, la guerrilla pidió disculpas a la comunidad por no acatar uno de los principios de la declaración de convivencia pacífica (entrevistas con pobladores, 2016).

No obstante, a diferencia de Samaniego donde el movimiento de resistencia civil mantuvo su cohesión frente a los actores armados, en Las Mercedes la unidad del movimiento de resistencia civil se fracturó rápidamente (entrevista con poblador, 2016). A finales de 2007, dos miembros de la comunidad fueron asesinados en el casco urbano y otro salió del país. El incidente causó zozobra en el pueblo, llevó al declive de Amigos por la paz y a la pérdida del apoyo de la comunidad, ya que muchos pobladores consideraron el incidente como una muestra del fracaso de la iniciativa (entrevistas con moradores y actores externos, 2014-2016). Luego de los asesinatos, la desunión de la comunidad fue aún más evidente. A partir de entonces muchos pobladores se alejaron de los líderes de la iniciativa, argumentando que los asesinatos fueron consecuencia de actividades en las cuales no debieron involucrarse. Los rumores sobre las muertes infundieron miedo en la población e hicieron que muchas personas dejaran de apoyar a Amigos por la paz y que la comunidad regresara a regirse por las reglas de juego impuestas por el grupo armado dominante que en aquel momento eran las FARC (entrevistas con pobladores y actores externos, 2014-2016). Según Ana Arjona (2015, pp. 186-187), la división hace improbable la resistencia en contra de los actores armados por al menos dos razones: primero, es difícil para la comunidad ponerse de acuerdo sobre las acciones a seguir; segundo, si algunos miembros de la comunidad deciden colaborar con algún grupo armado, puede ser peligroso para los demás miembros de la comunidad resistir.

Otro factor fundamental a tomar en cuenta es la participación en el movimiento. Según académicos como Ana Arjona (2014) y Oliver Kaplan (2013), la acción colectiva incrementa la capacidad de negociación de la población civil con los grupos armados y ofrece mayor protección. Igualmente, Chenoweth y Stephan (2011) consideran la participación masiva de la gente como una de las principales razones del éxito de las campañas 
de resistencia civil. En Samaniego, la participación de la comunidad fue significativa desde 1997, cuando se dieron las elecciones locales ganadas por Manuel Cuéllar hasta el año 2015. Entre 2009 y 2015 se dieron más de diez manifestaciones pacíficas, marchas y actividades similares en las cuales se involucraron miles de personas (Mouly et al., 2016, p. 140). Dicha participación se debió en cierta medida al gran número de organizaciones que tiene Samaniego, producto de un proceso organizativo de larga data: grupos de mujeres, jóvenes, niños, mototaxistas, comerciantes y campesinos, entre otros. Además, se usaron medios de comunicación locales, como radio y televisión, así como celulares y alto parlantes en vehículos para difundir las convocatorias a acciones de resistencia civil (Alcaldía de Samaniego, 2002, pp. 115-119). La organización de la población y su capacidad de convocatoria permitieron una amplia participación en acciones destacadas.

Al contrario, en Las Mercedes la participación en las movilizaciones organizadas en el año 2005 fue reducida (entrevistas con pobladores, 2014). En el año 2010 se realizaron marchas en solidaridad con siete policías que fallecieron en un ataque insurgente, pero no fueron específicas de Las Mercedes, sino que participó gente de todo el departamento de Norte de Santander en rechazo a la violencia ( $E l$ Tiempo, 2010). Solamente en 2013 la comunidad salió a protestar contra la detención de varios moradores de Las Mercedes, quienes eran acusados de colaborar con la guerrilla (La Opinión, 2013c; entrevistas con pobladores y miembros de organizaciones externas, 2016).

Durante el periodo de estudio la comunidad de Las Mercedes enfrentó principalmente tres obstáculos para lograr una participación masiva en las acciones de resistencia civil. El primero fue la deficiente infraestructura en telecomunicaciones que dificultó tanto la transmisión de decisiones como la participación en futuras reuniones por parte de la comunidad. Este tipo de comunicación es crucial para que una comunidad pueda quedarse al margen de la guerra, ya que permite una mayor participación de los miembros de la comunidad y permite a todos estar al tanto de las decisiones colectivas para actuar conforme a ellas (Anderson \& Wallace, 2013, p. 63). En 2015 solo cinco de las 47 veredas de Las Mercedes tenían acceso a telefonía celular y 
el acceso a Internet era limitado (Las Mercedes, 2015; entrevistas con pobladores y miembros de organizaciones externas, 2014-2016) ${ }^{7}$. La gente se comunicaba por medio de mensajes escritos sobre hojas de papel que amigos o familiares llevaban a otras personas. Esta forma de comunicación daba lugar a rumores e información errónea, y dificultaba las acciones conjuntas.

El segundo obstáculo fue el incipiente proceso organizativo de la comunidad, lo cual constituye una diferencia significativa con el caso de Samaniego. Si bien las juntas de acción comunal han funcionado en todas las veredas, no todas han sido efectivas según los pobladores. El único comité que ha funcionado en casi todas las juntas ha sido el comité de trabajo, encargado de realizar obras (Las Mercedes, 2015; entrevistas a pobladores, 2016). Además, por los flujos poblacionales (varios habitantes de Las Mercedes fueron a vivir en Cúcuta, mientras otros llegaron), este proceso organizativo tuvo poca continuidad. Para el año 2014 solo la mitad de los líderes de la iniciativa Amigos por la paz se encontraba todavía en Las Mercedes (entrevistas con pobladores y miembros de organizaciones externas, 2014). Por otro lado, aparte de las autoridades municipales electas en 2015, muchos gobiernos municipales anteriores no apoyaron el proceso de resistencia civil de Las Mercedes (entrevistas con actores externos y pobladores, 2014-2016). Esto también marca un contraste con el caso de Samaniego donde dos alcaldes dieron un impulso fuerte al proceso.

El tercer obstáculo fue el miedo y la desconfianza imperantes. El asesinato de los dos miembros de la comunidad, generó un ambiente de incertidumbre y temor que hizo que nadie se atreviera a dar continuidad a la iniciativa Amigos por la paz. En 2014 un grupo de líderes convocó a los habitantes de Las Mercedes a una reunión para elaborar el plan de desarrollo del corregimiento, pero muchos habitantes no asistieron por miedo y algunos dijeron haber recibido advertencias

\footnotetext{
En 2013 en Samaniego y Sardinata el número de suscriptores de Internet en relación a la población municipal era inferior a la media nacional con un índice de penetración inferior a $0,1 \%$. En el área rural de ambos municipios casi no había cobertura de telefonía fija y solo en pocas partes se podía hablar por celular (Criollo \& Castillo, 2013; Yanes, 2013).
} 
de parte de grupos armados (conversaciones informales con pobladores, 2014).

El liderazgo dentro de un movimiento de resistencia civil es otra característica esencial para el logro de sus propósitos. En su estudio de comunidades que consiguieron no involucrarse en la guerra, Anderson y Wallace (2013, pp. 52-53) encontraron que el liderazgo compartido -en contraposición al liderazgo individual - ofrece mayores oportunidades de continuidad y de apropiación de los procesos por parte de la población. En Samaniego el alcalde Cuéllar lideró la iniciativa de paz desde 1998 hasta el año 2000. De 2004 a 2007, el alcalde Harold Montúfar ejerció un liderazgo similar, impulsando diversas iniciativas, entre estas el pacto local de paz y una propuesta de desminado humanitario con el objetivo de quitar las minas colocadas por la guerrilla que dificultaban los movimientos de los pobladores del sector de la montaña $\mathrm{y}$ atentaban contra sus vidas. En los periodos inmediatamente posteriores a las administraciones de ambos líderes, las acciones de resistencia civil subsistieron, pero perdieron fuerza. En cambio, en el sector de la montaña hubo una mayor continuidad del proceso de resistencia civil por tener un liderazgo más colectivo, respaldado por la mayoría de moradores. Diversos líderes del sector de la montaña desempeñaron un papel notable en la organización de la población local desde los años ochenta e impulsaron acciones decisivas de resistencia civil desde 2008, cuando varias comunidades resultaron confinadas por la siembra de minas antipersonales en esta área (Mouly, et al., 2016; entrevistas con pobladores y miembros de organizaciones externas, 2014-2016). En Las Mercedes el liderazgo de la iniciativa Amigos por la paz no era tan "compartido" como en Samaniego. Nueve líderes impulsaron la iniciativa y consiguieron el respaldo de la comunidad para formular la declaración de convivencia pacífica (Las Mercedes, 2005) pero no lograron que la mayoría de la comunidad se involucrara de manera más contundente en el proceso de resistencia civil.

\section{Relación de la comunidad con los grupos armados}

La manera en que la comunidad interactúa con los grupos armados es igualmente importante para la eficacia de acciones de resistencia 
civil en contra de estos actores. El ELN, las FARC y los paramilitares (y luego grupos neoparamilitares), así como las fuerzas de seguridad del Estado (Policía y Ejército) han estado presentes en Samaniego. Varios pobladores se han integrado a estos grupos y algunos alcanzaron a tener altos cargos, lo que ha facilitado el establecimiento de canales de comunicación entre la población civil y estos grupos, sobre todo con la guerrilla y, en menor medida, con los paramilitares. Estos canales de comunicación permitieron consultar a los distintos grupos armados y socializar el pacto local de paz, el cual fue avalado públicamente por el ELN, las AUC y el Alto Comisionado para la Paz de Colombia (Lázaro, 2010; Restrepo, 2006) ${ }^{8}$. Además, antes de ser elegido alcalde, Montúfar fue detenido varias veces por distintos grupos armados no estatales que exigían explicaciones sobre su propuesta. Esto constituyó otra oportunidad para explicar y dar a conocer el pacto local de paz y el modelo de gestión que pretendía implementar si ganaba las elecciones. Estas oportunidades de diálogo pueden explicar por qué el pacto local de paz tuvo mayor éxito en la reducción de la violencia que la declaración de territorio de paz de 1998 que no se pudo socializar de la misma forma (Mouly et al., 2016).

Por otro lado, las interacciones históricas entre pobladores e insurgentes en el sector de la montaña ayudaron a la liberación de personas retenidas y permitieron a la población negociar con la guerrilla la administración de la justicia por parte de la comunidad en lugar de por parte de la guerrilla (entrevistas con pobladores, 2014-2016). Otro hito fue la negociación de medidas para facilitar la libre movilidad de la población afectada por minas antipersonales y el desminado de las principales vías de acceso al sector de la montaña. Durante la implementación de la política de seguridad democrática del expresidente Uribe, en el año 2008 se estableció una base militar en Samaniego, a unos diez minutos del centro, y las fuerzas armadas empezaron a

\footnotetext{
8 Véase declaración del ELN, recuperada de https://www.youtube.com/watch?v=W-HMjZUEH1s

Véase declaración de las AUC, recuperada de https://www.youtube.com/watch?v=DTo9DFC6keI Véase declaración del Alto Comisionado para la Paz durante su visita a Samaniego el 27 de diciembre de 2006, recuperada de https://www.youtube.com/watch?v=ZnlW7_5wi9s
} 
avanzar hacia el sector de la montaña, bastión de las guerrillas. Para protegerse e impedir el avance del Ejército, las guerrillas colocaron minas antipersonales en las principales vías de acceso al sector, que dejaron a la población local en situación de confinamiento por meses. Aunque la comunidad no logró la reubicación de la base militar fuera del casco urbano de Samaniego, negoció con el ELN y alcanzó un compromiso que consistió en poder salir del sector de la montaña a ciertas horas del día y tener dos teléfonos móviles para llamar a la guerrilla en caso de emergencia para que los dejaran salir del territorio sin lastimarlos. Posteriormente, consiguió un desminado parcial (Mejía, 2010; entrevistas con pobladores, 2014-2016). De la misma forma, varios pobladores del casco urbano negociaron directamente con la guerrilla para no pagar la llamada "vacuna" (entrevistas con pobladores, 2014).

Si bien la población civil siempre mantuvo canales de comunicación con los insurgentes, los esfuerzos de resistencia civil encontraron más obstáculos con la incursión paramilitar que pretendió poner fin al dominio de las guerrillas a inicios de los años 2000. Igualmente, la situación se complejizó durante los enfrentamientos entre FARC y ELN en el sector de la montaña entre 2007 y 2009 y cuando el Ejército empezó a ejercer mayor presión sobre los insurgentes. Ambos periodos se caracterizaron por un incremento de la violencia (Mouly, et al., 2016, p. 141). Mitchell y Hancock (2007) plantean que, cuando varios grupos armados se disputan un territorio, asegurar el respecto de un territorio de paz es un desafío porque cada actor en pugna puede considerar que las acciones de resistencia civil de la población están dando ventajas a sus enemigos (pp. 201-202).

En Las Mercedes también las frecuentes interacciones entre la población civil y el grupo armado dominante fueron clave para la eficacia de varias acciones de resistencia civil, incluyendo la influencia inicial de la declaración de convivencia pacífica sobre el modus operandi de los actores armados. La declaración fue adoptada después de un periodo de casi cinco años durante el cual las AUC retaron el control de las guerrillas. Al momento de la declaración, las FARC nuevamente eran el grupo dominante en Las Mercedes y se mantuvieron como tales 
durante casi todo el periodo de estudio, aunque en el año 2015 se replegaron a raíz de su declaración de cese al fuego unilateral en el marco de las negociaciones de paz (entrevistas con pobladores y miembros de organizaciones externas, 2016). Después de su desmovilización, fueron remplazadas por la guerrilla del EPL ( La Opinión, 2016; Prieto, 2017; Salgar, 2016). Al comienzo de la iniciativa de paz en 2005, la comunidad logró socializar con las FARC y otros actores armados y civiles su propuesta de mantenerse imparcial y no recurrir a la violencia. Así impuso a los grupos armados ciertas reglas de juego como no involucrar a la población civil en el conflicto armado. En un principio, las FARC y otros actores armados avalaron la propuesta, lo que contribuyó a reducir los abusos en contra de la población civil (entrevistas con pobladores, 2016; OEA, 2008). Según un excomandante de las FARC, su organización respetaba este tipo de propuestas de paz e imparcialidad (entrevista con excomandante de las FARC, 2016).

Sin embargo, los canales de comunicación con las FARC se rompieron a raíz de un cambio de comandante a nivel local y los rumores hicieron que dos miembros de la comunidad, acusados de colaborar con paramilitares, fueran asesinados a finales de 2007 (entrevistas confidenciales, 2014-2016). Con el asesinato, que tuvo lugar en el casco urbano, las reglas de la declaración de convivencia pacífica fueron violadas, lo que llevó al declive de la propuesta de Amigos por la paz y a la imposición de las reglas del grupo armado dominante. Dicho esto, varias fuentes coincidieron en que las causas más profundas del declive de la iniciativa fueron las divisiones internas de la comunidad y la falta de un liderazgo que generara la confianza necesaria en el proceso para negociar de forma conjunta con los grupos armados. Esta desunión podría haber permitido que estos grupos no vieran la iniciativa como algo de toda la comunidad y no respetaran las reglas (entrevistas con pobladores y miembros de organizaciones externas, 2014-2016). Solamente después del inicio de las negociaciones de paz con las FARC se entablaron nuevos diálogos con este grupo insurgente para recuperar el uso del parque central de Las Mercedes y organizar allí la fiesta anual del pueblo (entrevistas con pobladores, 2014-2016). Los esfuerzos de resistencia civil de la población alcanzaron un hito 
a fines de 2015 con la finalización de su plan de desarrollo, el cual recoge muchos puntos de la declaración de convivencia pacífica (Las Mercedes, 2015).

Vale la pena mencionar también que las frecuentes interacciones entre la población de Las Mercedes y las FARC resultaron en la estigmatización de la comunidad como colaboradora de la guerrilla y dificultaron el diálogo con las fuerzas de seguridad estatales, sobre todo con la Policía, después de su restablecimiento en el casco urbano al término del proceso de desmovilización de las AUC. Por este motivo varias personas de la comunidad fueron detenidas en 2013. Además, la reubicación del puesto de Policía en la esquina del parque central generó un conflicto entre la Policía y la comunidad, ya que la población argumentó que esto ponía en peligro a los vecinos, mientras la Policía planteó que trasladar la estación dejaría al pueblo bajo el control de la guerrilla y expondría a sus agentes. Esta tensión llevó a la población civil a optar por la vía judicial para lograr el traslado del puesto policial, lo que la distanció aún más de la Policía. En 2013 la Corte Suprema de Justicia de Colombia emitió un fallo a favor de los pobladores (La Opinión, 2013a), pero hasta inicios de 2016 la sentencia no se había implementado y los diálogos de la población con la Policía y las autoridades estatales no habían resuelto el problema (entrevistas con miembros de organizaciones externas, 2016).

\section{Rol de los actores externos}

El involucramiento de actores externos jugó un rol importante en ambos territorios. En Samaniego, la Red Nacional de Iniciativas Ciudadanas por la Paz y contra la Guerra (Redepaz) y la Pastoral Social de la Iglesia católica apoyaron el proceso de resistencia civil desde sus inicios. El municipio fue parte de un proyecto de la organización de la sociedad civil Redepaz que fomentó el intercambio de experiencias entre más de cien territorios de paz en Colombia como, por ejemplo, el de Mogotes, en el departamento de Santander (García Durán \& Sarmiento, 2015). Por este medio, muchos líderes locales recibieron entrenamiento en temas vinculados con la construcción de paz. Asimismo, el proyecto apoyó iniciativas locales de la sociedad civil y fomentó la organización de grupos 
de mujeres, jóvenes y niños que emprendieron varias acciones por la paz y contra la guerra. Esto contribuyó a la consolidación del movimiento de resistencia civil. Aun cuando el movimiento se debilitó con la terminación del periodo de Manuel Cuéllar, este se mantuvo gracias a los esfuerzos continuos de líderes de distintos sectores sociales a nivel local. De la misma forma, si bien la culminación de la gestión del alcalde Montúfar significó el fin del pacto local de paz, el movimiento de resistencia civil permaneció activo durante los gobiernos municipales posteriores y en el sector de la montaña cobró mayor impulso a raíz del confinamiento de varias comunidades por la siembra de minas antipersonales.

El proceso de resistencia civil del sector de la montaña se basó en un proceso organizativo de larga data. Sin embargo, se fortaleció cuando los líderes del sector empezaron a dar a conocer la situación de las comunidades confinadas a nivel nacional e internacional en el año 2008, y las comunidades del sector empezaron a recibir el apoyo de varias organizaciones externas (Garrido, Mouly \& Idler, 2016). En un principio, algunas organizaciones se juntaron para realizar una misión humanitaria interinstitucional al sector de la montaña para conocer las necesidades de las comunidades confinadas de primera mano (Centro Nacional de Memoria Histórica, 2015). Posteriormente, varias organizaciones externas, incluyendo agencias de Naciones Unidas y ONG internacionales y nacionales, apoyaron el proceso organizativo del sector de la montaña, abarcando la capacitación de líderes en derechos humanos, la conformación de un comité de derechos humanos, la elaboración de un plan de desarrollo y la constitución de un cabildo para garantizar la imparcialidad de la población en medio del conflicto armado (entrevistas con pobladores, actores externos y funcionarios del Estado, 2014-2016). El comité de derechos humanos en particular aumentó el poder de negociación de la población con los actores armados, sobre todo estatales, gracias al acompañamiento de varias organizaciones. Un entrevistado explicó que, cuando las fuerzas de seguridad estatales detenían injustamente a un poblador del sector de la montaña, el comité llamaba a su red de apoyo y gracias a esto soltaban a la persona detenida (entrevista con poblador, 2016). 
Por su parte, Las Mercedes ha sido un caso relativamente aislado que se benefició poco del intercambio con otras experiencias similares y del apoyo de organizaciones nacionales e internacionales. Aunque nunca fue parte de un proyecto de Redepaz, la comunidad recibió inicialmente el acompañamiento de la OEA y de la Iglesia católica, y posteriormente de agencias de Naciones Unidas, como la oficina del Alto Comisionado de Naciones Unidas para los Refugiados. El apoyo incluyó la capacitación de líderes, el respaldo a la iniciativa Amigos por la paz y la interlocución con instituciones estatales para fortalecer la presencia del Estado en la comunidad. No obstante, luego del fracaso de ella y frente a la poca presencia de organismos estatales que garantizaran la protección de la población civil y evitaran que esta regresara al control del grupo guerrillero dominante, los actores externos decidieron limitar su accionar hasta volver a encontrar condiciones propicias. Estas se dieron con el arranque del proceso de paz entre el Gobierno colombiano y las FARC a finales de 2012. En 2013, la comunidad pidió a varias organizaciones externas que acompañaran la marcha para pedir la liberación de los líderes detenidos por supuesta colaboración con la guerrilla. Aunque es difícil estimar el impacto de este acompañamiento, la marcha recibió cobertura en los medios locales y, a inicios de 2016, solo un líder permanecía detenido (entrevistas con pobladores y miembros de organizaciones externas, 2014-2016). Varias organizaciones también empezaron a apoyar un proceso participativo de diagnóstico de las necesidades de la comunidad que desembocó en la elaboración de un plan comunitario de desarrollo a finales de 2015 (Las Mercedes, 2015; Sánchez, 2015). De manera general, los actores armados han respetado las organizaciones externas, lo que posibilitó que la comunidad se reuniera nuevamente sin miedo y se organizara para enfrentar los efectos negativos de la guerra. Un excomandante de las FARC destacó los aportes de estas organizaciones al fortalecimiento de la organización comunitaria (entrevista con excomandante de las FARC, 2016). 


\section{Iniciativas locales de paz y construcción de paz a nivel nacional}

Las iniciativas locales de paz como los territorios de paz pueden influir positivamente en los procesos de paz a nivel nacional. Recíprocamente, las negociaciones de paz a nivel nacional pueden traer beneficios para los esfuerzos locales de paz, como en el caso de Las Mercedes. Después de su declive en 2007, el proceso de resistencia civil de Las Mercedes retomó fuerza con las negociaciones de paz entre el Gobierno colombiano y las FARC que arrancaron a finales del año 2012. Según varios pobladores de Las Mercedes, las negociaciones de paz constituyeron un contexto favorable para retomar el proceso iniciado en el año 2005 y obtener concesiones de parte de los grupos armados (entrevistas con pobladores, 2014-2016). A raíz de estas negociaciones de paz, se notó una mayor apertura al diálogo de parte de los mandos de las FARC (entrevista con miembro de organización externa, 2016). Con la intermediación del sacerdote, la comunidad logró recuperar el parque central como lugar público en las noches y organizar anualmente la fiesta patronal de Las Mercedes en este lugar, luego de un largo periodo sin poder hacerlo como consecuencia de la instalación de un puesto temporal de Policía en el parque central en 2007 (La Opinión, 2013b; entrevistas con pobladores y miembros de organizaciones externas, 2014-2016). Por otro lado, la marcha por la paz de noviembre de 2013 fue la primera de esta naturaleza después de varios años (La Opinión, 2013c). Ocurrió aproximadamente un año después del inicio de las negociaciones de paz con las FARC, cuando las partes habían alcanzado un acuerdo sobre el primer punto de la agenda, el tema agrario.

De manera general, los procesos de negociaciones de paz en Colombia estuvieron acompañados por un fortalecimiento de movimientos por la paz, como lo muestran los datos recopilados durante más de 25 años por el Centro de Investigación y Educación Popular - CINEP(García Durán, 2006). Este fue principalmente el caso durante los procesos de paz entablados por los gobiernos de los expresidentes Ernesto Samper y Andrés Pastrana, con un pico en 1998 cuando Samaniego fue declarado territorio de paz, y durante las negociaciones 
de paz entre el gobierno de Juan Manuel Santos y las FARC (CINEP, 2014, pp. 24-25).

El proceso de negociación del Gobierno colombiano con las AUC y su desmovilización posterior también resultaron favorables para las iniciativas locales de paz. En 2004 el Bloque Libertadores del Sur de las AUC aceptó el pacto local de paz propuesto por el alcalde de Samaniego cuando estaba negociando su desmovilización con el Gobierno nacional. De forma parecida, el inicio del proceso de resistencia civil en Las Mercedes coincidió con la desmovilización de los integrantes del Bloque Catatumbo de las AUC.

Algunos académicos plantean que los esfuerzos locales de paz pueden ocurrir independientemente del nivel nacional y que las experiencias acumuladas pueden influenciar el nivel nacional (Mitchell \& Hancock, 2012). En ese sentido, se considera fundamental prestar atención a las iniciativas locales que han fomentado la paz a través de sus propias estrategias y recursos como una alternativa a la política nacional de confrontación armada. En particular, el reconocimiento de iniciativas locales de paz en el discurso a nivel nacional puede generar una difusión de estos procesos en otras localidades afectadas por el conflicto armado en Colombia. Dado que los casos aquí presentados sugieren que el éxito de los territorios de paz se debe en parte al conocimiento de otras experiencias similares, la divulgación e intercambio de estas iniciativas pueden potenciarlas. Además, la inclusión de iniciativas locales de paz dentro del proceso nacional de construcción de paz garantiza una mayor apropiación del proceso por parte de las bases. Esta apropiación presenta retos pero es esencial para alcanzar una paz incluyente y sostenible a largo plazo (ej. Donais 2009).

\section{Conclusiones}

En este artículo comparamos dos experiencias de territorios de paz: Samaniego y Las Mercedes. En ambas localidades la violencia de los grupos armados estatales y no estatales llevó a la población a organizarse con el objetivo de mantener a la población e infraestructura civil fuera del conflicto violento. Estas experiencias dejaron entrever tres 
elementos clave para el éxito de iniciativas similares y confirmaron los factores identificados por Hancock y Mitchell (2007) y Anderson y Wallace (2013) para el éxito de territorios de paz. En ambos lugares la participación masiva de la población y el respaldo de la comunidad fueron esenciales para el éxito de las acciones de resistencia civil. No obstante, en Las Mercedes se presentaron mayores dificultades debido a la poca cohesión del movimiento de resistencia civil, la falta de confianza, la cultura de participación incipiente, la comunicación reducida y la poca representatividad de los líderes de la iniciativa. En cambio, en Samaniego el movimiento de resistencia civil fue más consolidado, con liderazgos reconocidos y la participación y compromiso de gran parte de la población, lo que le permitió alcanzar más logros. Las estrategias locales de resistencia civil se fundamentaron en la organización comunitaria, la comunicación y los principios de no-violencia e imparcialidad.

Las relaciones entre comunidad y actores armados también jugaron un papel central. Varios miembros de los grupos armados que operaban en Samaniego eran oriundos de la zona, tenían familiares o conocidos allí, lo que facilitó el diálogo entre la comunidad y estos actores, y contribuyó a la eficacia de varias acciones de resistencia civil. En Las Mercedes las interacciones entre la comunidad y los grupos insurgentes fueron frecuentes, pero los canales de comunicación entre la población y el grupo insurgente dominante se rompieron por un cambio de comandante, lo que dificultó la interlocución. Mientras las interacciones entre comunidad y grupos armados no estatales fueron útiles para lograr un mayor respeto de la población civil por parte de estos grupos, distanciaron a estas comunidades de los actores armados estatales, sobre todo en el caso de Las Mercedes. Esto redujo la posibilidad de que los pobladores de Las Mercedes obtuvieran concesiones de parte de la Policía e hizo que escogieran la vía judicial como el medio principal para alcanzar la reubicación del puesto de Policía.

El apoyo de actores externos puede potenciar los procesos de resistencia civil e incidir en el nivel de organización de un movimiento porque ayuda a que la población se empodere de sus derechos y acceda a recursos. También puede ayudar a que las demandas de la 
población civil local sean mejor atendidas por los actores armados, sobre todo estatales. Sin embargo, puede ser problemático si los actores externos impulsan estas iniciativas sin que la comunidad se apropie genuinamente de las mismas.

El estudio también reveló que varios de los factores mencionados por Mitchell y Hancock y Anderson y Wallace son complejos y dependen del contexto local. Por ejemplo, la capacidad de interlocución de la comunidad con los actores armados puede variar en relación a los distintos actores armados y depende de la dinámica local del conflicto, incluyendo el poder de cada actor armado en el territorio y la relación de la población con cada actor. Por otro lado, esta capacidad de interlocución puede terminar repentinamente, como ocurrió en Las Mercedes, cuando se cortaron los canales de comunicación entre la comunidad y las FARC por un cambio de mando. De la misma forma, las características del movimiento de resistencia civil son producto tanto de la agencia como de la estructura. Así, en Samaniego, donde existía un proceso organizativo de larga data, fue más fácil asegurar la participación y la unidad en el movimiento de resistencia civil. Además, el movimiento se fue fortaleciendo a lo largo del tiempo, facilitando cada vez más la participación y la cohesión. La dinámica del conflicto no solo incide en los tres factores identificados, sino en las mismas estrategias de los actores. El caso de Las Mercedes lo ejemplifica. A pesar de que inicialmente la comunidad adoptó una estrategia similar a la de la población de Samaniego, planteando ciertas reglas de juego a los actores armados presentes en su territorio mediante una declaratoria, y tuvo éxito, luego las circunstancias cambiaron y la comunidad adaptó su estrategia al nuevo contexto en el que se encontraba.

Por otra parte, coincidimos con autores como Bo Wirmark, quien planteó que para que un movimiento no-violento fuese exitoso debía tener raíces en la estructura de la comunidad y de esta estructura dependían los métodos y estrategias más efectivos para alcanzar sus propósitos (Wirmark citado en Hallward \& Norman, 2015, p. 27). El caso del movimiento del sector de la montaña, el cual decidió conformar un cabildo como estrategia de resistencia frente a los actores 
armados, es ilustrativo. Si bien esta iniciativa se benefició del apoyo de organizaciones externas, surgió con base en la realidad local y el proceso organizativo de la población del sector que, a pesar de no ser indígena en su gran mayoría, había creado sus propias estructuras de gobernanza frente a la ausencia del Estado.

Finalmente, a nivel nacional, la política de seguridad democrática priorizó la militarización como vía para la resolución del conflicto armado y produjo más violencia en lugares periféricos como Samaniego y Las Mercedes. En cambio, los procesos de paz favorecieron las experiencias de resistencia civil a nivel local. Así las negociaciones de paz entre el gobierno del presidente Santos y las FARC fomentaron un ambiente propicio para el desarrollo de iniciativas locales de paz en Samaniego y Las Mercedes. Estas iniciativas pueden aportar a la paz nacional generando un cambio de discurso y una mayor apropiación del proceso. Es importante seguir investigando los procesos de resistencia civil en contextos de conflicto armado puesto que otras iniciativas no han sido analizadas y podrían facultar un mejor entendimiento de la incidencia de los factores identificados en los casos de Samaniego y Las Mercedes en el éxito o fracaso de estos procesos.

\section{Agradecimientos}

Agradecemos el apoyo valioso de Annette Idler en el proyecto de investigación y en la fase inicial de elaboración de este artículo. También agradecemos los comentarios útiles de los evaluadores.

\section{Referencias}

Alcaldía de Samaniego. (2002). Plan de desarrollo municipal: Samaniego por el camino de la prosperidad, 2012-2015.

Alther, G. (2006). Colombian peace communities: The role of NGOs in supporting resistance to violence and oppression. Development in Practice, 16 (3/4), 278-291.

Anderson, M. B., \& Wallace, M. (2013). Opting out of war: Strategies to prevent violent conflict. Boulder, CO: Lynne Rienner. 
Arjona, A. (2014). Civilian resistance to rebel governance. HiCN Working Paper 170. Brighton: Households in Conflict Network, Institute of Development Studies, Universidad de Sussex.

Arjona, A. (2015). Civilian resistance to rebel governance. En A. Arjona, N. Kafir \& Z. Mampilly (Eds.), Rebel governance in Civil War (pp. 180202). Nueva York: Cambridge University Press.

Centro Nacional de Memoria Histórica. (2015). Confinados: El caso de Samaniego.

CINEP. (2014). Estrategias y retos políticos para la paz: Recomendaciones para el sector politico y la sociedad civil. Informe especial.

Chenoweth, E., \& Cunningham, K. G. (2013). Understanding nonviolent resistance: An introduction. Journal of Peace Research, 50(3), 271-276. doi: $10.1177 / 0022343313480381$

Chenoweth, E., \& Stephan, M. J. (2011). Why civil resistance works: The strategic logic of nonviolent conflict. Nueva York: Columbia University Press.

Criollo, H., \& Castillo, A.M. (16 de mayo de 2013). Perfilproductivo. Municipio Samaiego-Nariño. Mintrabajo; Red Ormet \& PNUD.

Diario del Sur. (22 de noviembre de 1997a). Samaniego respaldó a sus elegidos. Diario del Sur.

Diario del Sur. (25 de noviembre de 1997b). Un llamado para que se respete voluntad popular. Diario del Sur.

Diario del Sur (23 de enero de 1998a). Declaran a Samaniego territorio de paz y libertad. Diario del Sur.

Diario del Sur. (26 de enero de 1998b). Informe Samaniego: imágenes de paz. Diario del Sur.

Diario del Sur. (26 de enero de 1998c). Samaniego cambió rostro de guerra por el de la paz. Diario del Sur.

Donais, T. (2009). Empowerment or imposition? Dilemmas of local ownership in post-conflict peacebuilding processes. Peace \& Change, 4(1). doi: 10.1111/j.1468-0130.2009.00531.x

El Tiempo. (22 de noviembre de 1997a). Samaniego le exige respeto a la guerrilla, El Tiempo. Recuperado de http://www.eltiempo.com/archivo/ documento/MAM-666460

ElTiempo. (24 de noviembre de 1997b). Samaniego quiere decidir su suerte, ElTiempo. Recuperado de http://www.eltiempo.com/archivo/documento/MAM-670076 
El Tiempo. (21 de noviembre de 1997c). Todo un pueblo refrenda hoy elección de sus autoridades, El Tiempo. Recuperado de http://www. eltiempo.com/archivo/documento/MAM-662350

El Tiempo. (30 de junio de 2010). Marchas simultáneas contra el terrorismo en varios municipios nortesantandereanos, El Tiempo. Recuperado de http://www.eltiempo.com/archivo/documento/CMS-7783480

García, A., \& Llinás, G. (2012). Los programas de desarrollo y paz (PDP) de Colombia: una apuesta innovadora por el desarrollo y la paz en medio del conflicto armado. En A. Rettberg (Ed.), Construcción de paz en Colombia (pp. 273-304). Bogotá: Universidad de los Andes.

García Durán, M. (2006). Movimiento por la paz en Colombia 1978-2003. Bogotá: CINEP.

García Durán, M., \& Sarmiento, F. (2015). Demining in micoahumado: From civil resistance to local negotiation with the ELN. En S. Haspeslagh \& Z. Yousuf (Eds.), Local engagement with armed groups: In the midst of violence. accord insight 2 (pp. 21-26). Londres: Conciliation Resources. (pp. 21-26).

Garrido, M. B., Mouly, C., \& Idler, A. (2016). Jiu-jitsu en contexto de conflicto armado: el poder de la resistencia no violenta. Ciudad paz-ando, 9(2), 155-167.

Hallward, M. C., \& Norman, J. M. (Eds.). (2015). Understanding Nonviolence. Cambridge: Polity.

Hancock, L., \& Mitchell, C. (Eds.). (2007). Zones of peace. Bloomfield, CT: Kumarian Press.

Hernández, E. (2004). Resistencia civil artesana de paz: experiencias indigenas, afro descendientes y campesinas. Bogotá: Editorial Pontificia Universidad Javeriana.

Hernández, E. (2012). Intervenir antes de que anochezca: mediaciones, intermediaciones y diplomacias no violentas de base social en el conflicto armado. Bogotá: La Bastilla.

Idler, A., Garrido, M. B., \& Mouly, C. (2015). Peace territories in Colombia: Comparing civil resistance in two war-torn communities. Journal of Peacebuilding \& Development, 10(3), 1-15. doi: 10.1080/15423166.2015.1082437

Idler, A., Mouly, C., \& Garrido, M. B. (por publicar). Between shadow citizenship and civil resistance: Shifting local orders in a Colombian 
war-torn community. En L. Hancock \& C. Mitchell (Eds.), Local PeaceBuilding and Legitimacy. Londres: Routledge.

Jaramillo, S. (2014). La paz territorial. Presentación en la Universidad de Harvard, Cambridge, MA, Estados Unidos, 13 de marzo.

Kaplan, O. (2013). Nudging armed groups: How civilians transmit norms of protection. Stability: International Journal of Security \& Development, 2(3), 1-18.

La Opinión. (12 de julio de 2013a). Corte ordena traslado de la estación de policía de Las Mercedes, La Opinión.

La Opinión. (12 de noviembre de 2013b). En Las Mercedes se vive con el Credo en la boca, La Opinión.

La Opinión. (6 de noviembre, 2013c). Los mercedeños se volcaron a las calles, La Opinión.

La Opinión. (24 de febrero de 2016). Sardinata: tres asesinatos en un día, La Opinión. Recuperado de http://www.laopinion.com.co/judicial/ sardinata-tres-asesinatos-en-un-dia-107397

Las Mercedes. (2005). Declaración de convivencia pacífica: Las Mercedes, Sardinata, Norte de Santander, adoptada por la comunidad de Las Mercedes el 19 de diciembre.

Las Mercedes. (agosto de 2015). Plan comunitario: corregimiento Las Mercedes. Cúcuta.

Lázaro, J. L. (2010). Iniciativas locales de paz: el caso del gobierno municipal de Samaniego 2004-2007. En C. de Gamboa Tapias (Ed.), El tránsito hacia la paz: de las herramientas nacionales a las locales (pp. 241-261). Bogotá: Editorial Universidad del Rosario.

Lederach, J. P. (1997). Building peace: Sustainable reconciliation in divided societies. Washington, D.C.: United States Institute of Peace Press.

Mejía, J. E. (15 de febrero de 2010). Con valor, campesinos de Samaniego le hacen frente a la guerra. Semana. Recuperado de http://www.semana. $\mathrm{com} /$ nacion/problemas-sociales/articulo/con-valor-campesinossamaniegohacen-frente-guerra/112985-3

Mitchell, C., \& Allen Nan, S. (1996). Local peace zones as institutionalized conflict. Peace Review: A Journal of Social Justice, 9(2), 159-162.

Mitchell, C., \& Hancock, L. (2007). Local zones of peace and a theory of sanctuary. En L. Hancock \& C. Mitchell (Eds.), Zones of peace (pp. 189221). Bloomfield, CT: Kumarian Press. 
Mitchell, C., \& Hancock, L. (Eds.). (2012). LocalPeacebuilding and national peace: Interaction between grassroots and elite processes. Nueva York: Continuum. Mitchell, C., \& Rojas, C. (2012). Against the stream: Colombian Zones of peace under democratic security. En C. Mitchell \& L. Hancock (Eds.), Local peacebuilding and national peace: Interaction between grassroots and elite processes (pp. 39-67). Londres: Continuum.

Montúfar, H. (Ed.). (2007). Árbol de la waycosidad 3. Pasto: Districomputo.

Mouly, C., Garrido, M. B., \& Idler, A. (2016). How peace takes shape locally: The experience of civil resistance of Samaniego in Colombia. Peace \& Change, 41(2), 129-166. doi: 10.1111/pech.12184

Mouly, I. \& Garrido, M. B. (2015). Zones of Peace in Colombia's Borderlands. International Journal of Peace Studies, 20(1).

OEA. (2008). Iniciativas comunitarias: Cúcuta [video]. Recuperado de https:// www.youtube.com/watch?v=vTYGCAtrIGw.

Peñaloza, A. (30 de enero de 2006). Sardinata se armó de valor. El Tiempo. Recuperado de http://www.eltiempo.com/archivo/documento/ MAM-1900106

Prieto, J. (23 de febrero de 2017). Las 10 razones por las que el EPL es un problema que se le creció al Gobierno. La silla vacía. Recuperado de http:/ / lasillavacia.com/historia/las-10-razones-por-las-que-el-eples-un-problema-que-se-le-crecio-al-gobierno-59861

Restrepo, J. D. (2 de mayo de 2006). Samaniego le apuesta a Pacto Local de Paz, Semana. Recuperado de http://www.semana.com/on-line/ articulo/samaniego-apuesta-pacto-local-paz/78661-3

Rojas, C. (2004). The people's peace processes: Local resistance processes and the development of a "zone of peace" in Colombia. Reflexion Política, 6(11), 70-87.

Rojas, C. (2007). Islands in the stream: A Comparative analysis of zones of peace within Colombia's civil war. En L. Hancock \& C. Mitchell (Eds.), Zones of peace (pp. 71-89). Bloomfield, CT: Kumarian Press.

Salgar, D. (16 de agosto de 2016). La comunidad que espera a las FARC en el Catatumbo, El Espectador. Recuperado de http://colombia2020. elespectador.com/territorio/la-comunidad-que-espera-las-farc-enel-catatumbo

Sánchez, H. (22 de noviembre de 2015). Habitantes de Las Mercedes construyeron su "plan de vida" hacia el desarrollo. La Opinión. 
Sanford, V. (2003). Eyewitness: Peacebuilding in a war zone: The case of Colombian peace communities. International Peacekeeping, 10(2), 107-118. Schock, K. (2013). The practice and study of civil resistance. Journal of Peace Research, 50(3), 277-290. doi: 10.1177/0022343313476530

Yanes, S. J. (2013). Perfil productivo. Municipio Sardinata. Mintrabajo; Red Ormet \& PNUD. 


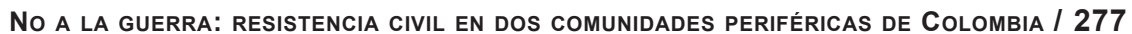

\section{Anexo 1}

Entrevistas realizadas en Samaniego y Las Mercedes

\begin{tabular}{|l|c|c|}
\hline \multicolumn{1}{|c|}{ Entrevistados } & Samaniego & Las Mercedes \\
\hline exfuncionarios del estado & 19 & 10 \\
\hline exmiembros de organismos internacionales & 11 & 5 \\
\hline expobladores & 20 & 13 \\
\hline exactores armados & 9 & 2 \\
\hline $\begin{array}{l}\text { exmiembros de organizaciones de la sociedad } \\
\text { civil colombiana }\end{array}$ & 30 & 10 \\
\hline
\end{tabular}

